\title{
Synthesis of Rearranged $\mathrm{N}$-Tosyl $\mathrm{Aza}$-Baylis-Hillman Adducts under Acidic Conditions Catalyzed by $\mathrm{CH}_{3} \mathrm{SO}_{3} \mathrm{H}$ or Montmorillonite $\mathrm{K} 10$
}

\author{
Hoo Sook Kim, Hyun Seung Lee, and Jae Nyoung Kim \\ Department of Chemistry and Institute of Basic Science, Chomam National University, Gwangiu 500-757, Korea \\ E-mail: kiminachonnamackr \\ Received February 7, 2009, Accepted February 15, 2009
}

Key Words: Methanesulfonic acid. Montmorillonite K10. Az $q$-Baylis-Hillnan adducts

The Baylis-Hillman reaction and its aza-version have made great progress since the original Baylis-Hillman reaction was first reported in 1972. 'We and other research groups have used extensively the aza-Baylis-Hillman adducts and their rearranged derivatives for the synthesis of many heterocyclic compounds. ${ }^{1-3}$ Usually the rearranged N-tosyll aza-BaylisHillman adduct 3 a has been prepared from $N$-tosyl $a z a-B a y l i s-$ Hillman adduct 1a or Baylis-Hillman acetate 1b under the influence of $\mathrm{K}_{2} \mathrm{CO}_{3}$ in DMF as in Scheme $1^{2,3}$ The reported stereochemistry of the product $3 \mathbf{a}$ is predominantly $E^{2.3}$ The synthesis of the corresponding rearranged N-tosyl azaBaylis-Hillman adduct having nitrile moiety has not been reported.

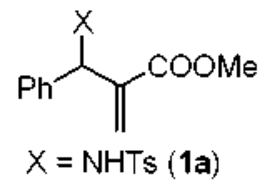
$X=O A C(1 b)$
During our recent studies on the radical cyclization and Heck type cyclization with modified Baylis-Hillnian adducts, ${ }^{4.5}$ the $Z$-form of rearranged $N$-tosyl $a z a$-Baylis-Hillman adduct was required. However. there was no precedent method for the synthesis of $Z$-form of rearranged $N$-toșyl aza-BaỵlisHillman adducts in appreciable anounts. Thus we decided to examine the preparation of these compounds under acidic conditions.

Actually, the reaction of 1a and tosylamide (2a) under basic conditions (DMF, $\mathrm{K}_{2} \mathrm{CO}_{3}$ ) produced $E$-form $(\mathbf{3 a}-E$ ) selectively (73\%) together with only $6 \%$ of $Z$-isomer as in Table 1 (entry 1). as mentioned above ${ }^{2,2}$ The reaction of Baylis-Hillman acetate $\mathbf{1 b}$ and $\mathbf{2 a}$ gave also similar results (entry 2).

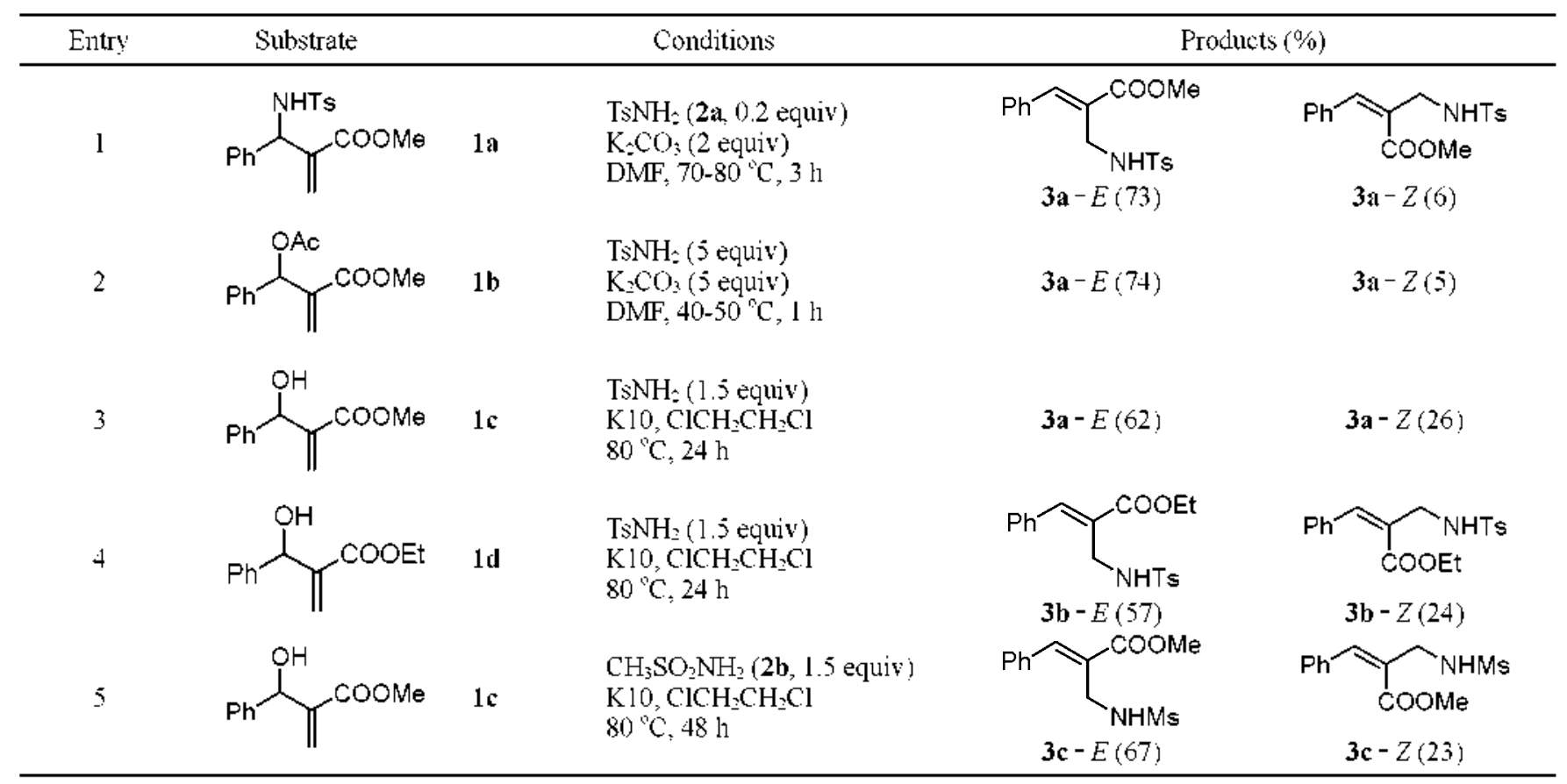

Scheme 1

Table 1. Conversion of Baylis-Hillman adducts into rearranged N-tosyl aza-Baylis-Hillman adducts 
Whereas, the reaction of Baylis-Hillman adduct $1 \mathrm{c}$ and $2 \mathrm{a}$ in the presence of montmorilonite K10 (entry 3$)^{6}$ produced appreciable amounts of desired 3a- $Z$ ( $26 \%$ ). Under such acidic conditions the reaction of ethyl ester $\mathbf{1 d}(\mathrm{entry} 4$ ) and the reaction between 1c and $\mathrm{CH}_{3} \mathrm{SO}_{2} \mathrm{NH}_{2}(\mathbf{2 b})$ also showed similar results (entry 5).

Encouraged by the results we examined the possibility for the synthesis of $Z$-isomer in an increased yield under various acidic conditions with ethyl ester $1 \mathbf{d}$ as a model substrate. and the results are summarized in Table 2. As shown the use of montmorillonite $\mathrm{K} 10$ showed the formation of $3 \mathbf{b}-Z$ in appreciable amounts (19-26\%. entries $1-3)$. When we stopped the reaction in short time $(2 \mathrm{~h})$, we obtained desired compound $3 \mathbf{b}-Z$ in $26 \%$ yield and $a z a$-Baylis-Hillman adduct 1e was isolated in $21 \%$ (entry 1). The compound le was converted completely to $\mathbf{3 b}-E$ and $\mathbf{3 b}-Z$ for $24 \mathrm{~h}$ refluxing. however, the ratio of $\mathbf{3 b}-Z / \mathbf{3 b}-E$ was decreased slightly (entry 2 ). Increasing reaction time decreased the ratio of $3 \mathbf{b}-Z / \mathbf{3} \mathbf{b}-E$ (entries $2-3$ ). Methanesulfonic acid showed somewhat higher catalytic activity than the use of $\mathrm{KlO}$ (entries $4-7$ ), however. the selectivity for $3 \mathbf{b}-Z$ was not improved. The use of sulfuric acid was not satisfactory (entry 8). From the results. the conditions of entry 2 turned out to be the best choice for the preparation of $3 \mathrm{~b}-Z$ although the yield is still low (24\%).

As a next trial, we examined the synthesis of rearranged N-tosyl aza-Baylis-Hillman adduct of nitrile derivative $\mathbf{3 d}$ (Table 3). We obtained the product $3 d$ in only $40 \%$ yield as an inseparable $E Z Z$ mixture from the reaction of $\mathbf{1 f}$ under basic

Table 2. Optimization of reaction conditions

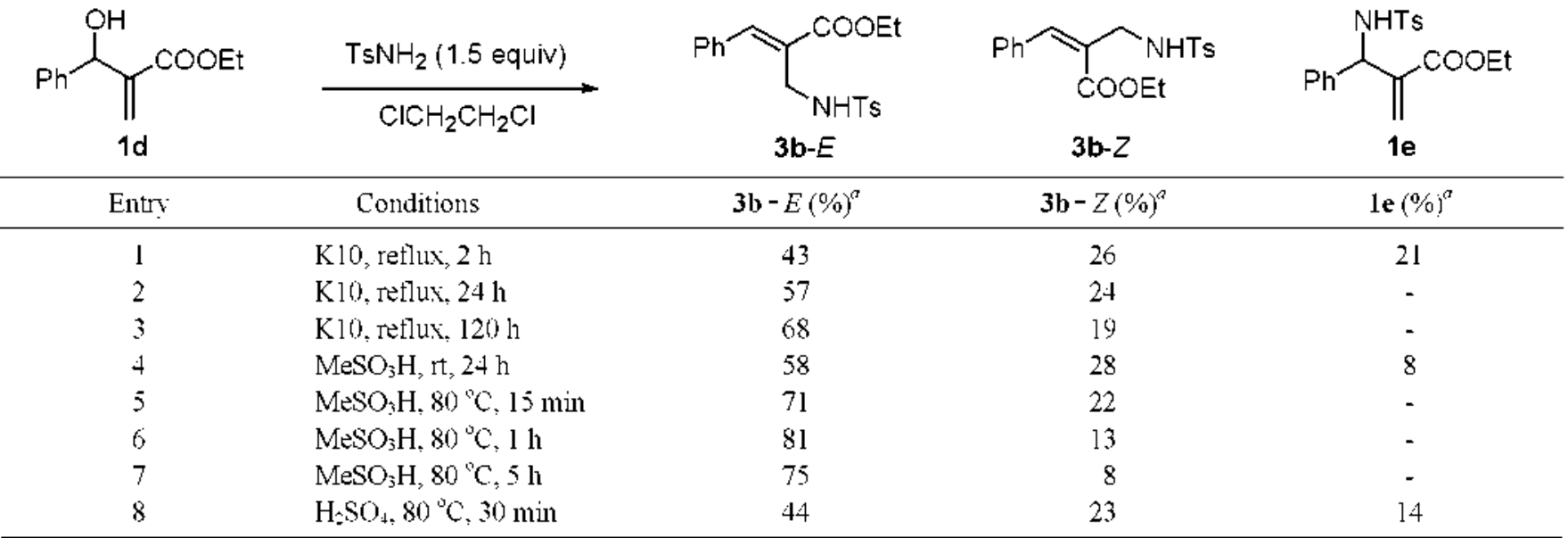

Isolated vield.

Table 3. Conversion of Baylis-Hillnan adducts into rearranged $N$-tosyl aza-Baylis-Hillman adducts

Entry Substrate

$\overline{{ }^{a}} E Z Z$ was not separated and the ratio was determined based on ${ }^{\mathrm{H}} \mathrm{H}$ NMR spectrum. ${ }^{\mathrm{t}} \mathrm{Three}$ stereoisomers $(E, E: E, Z: Z, Z)$ were mived together. Tot detected. "Pure isomer $(Z Z Z)$ was isolated in $7^{\circ}$. vield. "The compound $\mathbf{3 d}-Z$ was contaminated witl unreacted $\mathbf{l h}$ 
conditions (entry 1). The separation of $E$ and $Z$ isomers was impossible and we confirmed the ratio by ${ }^{l} \mathrm{H} N \mathrm{NR}$ as $E: Z=$ $2: 3$. A bis-adduct + was also obtained in $10 \%$ yield. ${ }^{8}$ The synthesis of $\mathbf{3 d}$ from the reaction of Baylis-Hillman acetate $\mathbf{~} \mathbf{g}$ and tosylamide was not successfil due to the formation of 4 as the major product even in the presence of excess amounts $(5.0$ equiv) of tosylamide in order to reduce the formation of 4 .

However. to our delight. we obtained $3 d-Z$ in $69 \%$ isolated yield when we carried out the reaction under acidic conditions with Baylis-Hillman adduct $1 \mathrm{~h}$ under the influence of $\mathrm{MeSO}_{3} \mathrm{H}$ (entry 3 ). It is interesting to note that the corresponding $E$-isomer was not formed even in trace amounts. In the reaction mixture we observed the formation of bis-adduct $t$ (7\%) and the stereochemistry of this compound was also found to be as $Z: Z$. Similarly $3 \mathrm{e}-Z$ was synthesized in good yield $(71 \%)$ under same acidic conditions from $1 \mathrm{~h}$ and $\mathrm{CH}_{3} \mathrm{SO}_{2} \mathrm{NH}_{2}$ (entry 4). The use of $\mathrm{K} 10$ in this case was found to be less effective than the use of K10 (entry 5 ).

In summary. we examined the synthesis of $N$-tosyl azaBaylis-Hillman adducts from the reaction of various BaylisHillman adducts and tosy lamide. We obtained $Z$-isomers up to $26 \%$ for the Baylis-Hillman adducts having ester moiety and $71 \%$ for the Baylis-Hillman adducts having nitrile moiety.

\section{Experimental Section}

Typical procedure for the synthesis of $3 \mathrm{a}$. A solution of 1c (192 $\mathrm{mg}, 1 \mathrm{mmol}$ ), TsNHz (257 $\mathrm{mg} .1 .5 \mathrm{mmol}$ ). and montmorillonite $\mathrm{K} 10$ (Aldrich. $550 \mathrm{mg}$ ) in 1.2-dichloroethane (5 $\mathrm{mL}$ ) was heated to reflux for $24 \mathrm{~h}$. After filtration of the reaction mixture. removal of solvent. and column chromatographic purification process (hexanes/EtOAc/ $\mathrm{CH}_{2} \mathrm{Cl}_{3}$. $6: 1: 3)$ we obtained $3 \mathrm{a}-E(21+\mathrm{mg}, 62 \%)$ and $3 \mathrm{a}-Z(90 \mathrm{mg}$. $26 \%$ ). The other compounds were synthesized analogously

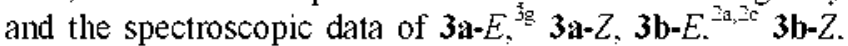
$3 \mathbf{c}-E$. and $\mathbf{3 c}-Z$ are as follows

Compound 3a- $E$ : ${ }^{39} 62 \%$ : white solid. mp $109-111{ }^{\circ} \mathrm{C}$; IR (film) 3263. 1705. 1326. $1161 \mathrm{~cm}^{-1}:{ }^{1} \mathrm{H} \mathrm{NMR}\left(\mathrm{CDCl}_{3}, 300\right.$ $\mathrm{MHz}) \delta 2.43$ (s. 3H). 3.76 (s.3H). 3.96 (d. $J=7.0 \mathrm{~Hz}, 2 \mathrm{H}$ ). $5.18(\mathrm{t} . J=7.0 \mathrm{~Hz} .1 \mathrm{H}) .7 .27(\mathrm{~d}, J=8.0 \mathrm{~Hz}, 2 \mathrm{H}), 7.38-7.40(\mathrm{~m}$, $5 \mathrm{H}) .7 .67(\mathrm{~d} . J=8.0 \mathrm{~Hz} .2 \mathrm{H}) .7 .75(\mathrm{~s} .1 \mathrm{H}):{ }^{13} \mathrm{C} \mathrm{NMR}\left(\mathrm{CDCl}_{3}\right.$. $75 \mathrm{MHz}) \delta 21.49 .40 .53 .52 .27,126.42 .127 .24,128.74$. $129.47 .129 .50,129.62,133.86,136.46,143.45 .143 .48 .167 .67$.

Compound 3a-Z: 26\%; colorless oil; IR (film) 3275, 1714. $1327.1160 \mathrm{~cm}^{-1}$ : ${ }^{1} \mathrm{H}$ NMR (CDCl. $\left.300 \mathrm{MHz}\right) \delta 2.36$ (5. $\left.3 \mathrm{H}\right)$, $3.56(\mathrm{~s} .3 \mathrm{H}) .3 .93(\mathrm{~d} . J=6.0 \mathrm{~Hz}, 2 \mathrm{H}), 5.21(\mathrm{t} . J=6.0 \mathrm{~Hz} . \mathrm{lH})$. $6.84(\mathrm{~s}, 1 \mathrm{H}) .7 .09-7.10(\mathrm{~m}, 2 \mathrm{H}), 7.25-7.29(\mathrm{~m} .5 \mathrm{H}) .7 .75(\mathrm{~d}, J$ $=8.0 \mathrm{~Hz} .2 \mathrm{H}):{ }^{13} \mathrm{C} \mathrm{NMR}\left(\mathrm{CDCl}_{3}, 75 \mathrm{MHz}\right)$ o 21.40 .47 .47 . 51.68. 127.14. 127.44, 127.93, 128.50, 128.56. 129.67. 134.75. $137.39 .139 .52,143.44,167.73$

Compound 3b-E: ${ }^{-2 c} 57 \%$ : white solid mp $112-11+{ }^{\circ} \mathrm{C}:{ }^{1} \mathrm{H}$ NMR $\left(\mathrm{CDCl}_{3} .300 \mathrm{MHz}\right) \delta 1.30(\mathrm{t}, J=7.5 \mathrm{~Hz}, 3 \mathrm{H}) .2 .42$ (s. $3 \mathrm{H}) .3 .95(\mathrm{~d} . J=6.5 \mathrm{~Hz}, 2 \mathrm{H}) .+2 \mathrm{l}(\mathrm{q} . J=7.5 \mathrm{~Hz}, 2 \mathrm{H}) .5 .4 \mathrm{l}(\mathrm{t}$. $J=6.5 \mathrm{~Hz} .1 \mathrm{H}) .7 .27(\mathrm{~d}, J=8.0 \mathrm{~Hz}, 2 \mathrm{H}) .7 .37-7.41(\mathrm{~m}, 5 \mathrm{H})$, 7.67 (d. $J=8.0 \mathrm{~Hz}, 2 \mathrm{H}) .7 .74$ (s, $1 \mathrm{H})$.

Compound 3b-Z: $24 \%$ : colorless oil: IR (film) $3277,1705$. $1328,1161 \mathrm{~cm}^{-1} ;{ }^{l} \mathrm{H} \mathrm{NMR}\left(\mathrm{CDCl}_{3}, 300 \mathrm{MHz}\right)$ ò $1.02(\mathrm{t}, J=7.0$ $\mathrm{Hz}, 3 \mathrm{H}) .2 .35(\mathrm{~s} .3 \mathrm{H}) .3 .92(\mathrm{~d}, J=6.5 \mathrm{~Hz}, 2 \mathrm{H}) .4 .0 \mathrm{I}(\mathrm{q} . J=7.0$
Hz. $2 \mathrm{H}$ ), 5.41 (br s. $1 \mathrm{H}), 6.8+(\mathrm{s}, 1 \mathrm{H}) .7 .08-7.10$ (n. $2 \mathrm{H})$, $7.23-7.26(\mathrm{~m}, 5 \mathrm{H}), 7.75(\mathrm{~d}, J=8.5 \mathrm{~Hz}, 2 \mathrm{H}) .{ }^{13} \mathrm{C} \mathrm{NMR}\left(\mathrm{CDCl}_{3}\right.$. $75 \mathrm{MHz}) \delta \delta 13.48,21.33,47.30 .60 .83,127.06,127.73$. 127.99. 128.28, 128.42, 129.58, 134.92. 137.38, 139.00, 143.28. 167.27.

Compound $3 \mathrm{C}-E$ : $67 \%$ : white solid, $m p$ 77.78 ${ }^{\circ} \mathrm{C}$; IR (film) $3283,1709,1322,1153 \mathrm{~cm}^{-1}:{ }^{1} \mathrm{H}$ NMR $\left(\mathrm{CDCl}_{3}, 300 \mathrm{MHz}\right) \delta$ $2.94(\mathrm{~s}, 3 \mathrm{H}), 3.87(\mathrm{~s}, 3 \mathrm{H}), 4.18(\mathrm{~d} . J=6.3 \mathrm{~Hz}, 2 \mathrm{H}), 4.95(\mathrm{t} . J=$ $6.3 \mathrm{~Hz} .1 \mathrm{H}) .7 .37-7.48(\mathrm{~m}, 5 \mathrm{H}), 7.89(\mathrm{~s}, 1 \mathrm{H}) ;{ }^{13} \mathrm{C}$ NMR $\left(\mathrm{CDCl}_{2}, 75 \mathrm{MHz}\right) \delta$ 40.08, 40.38, 52.46, 126.87, 128.92 . 129.36. 129.71. 133.78.143.68, 167.73

Compound 3c-Z: 23\%: colorless oil: IR (film) 3293,1716 .

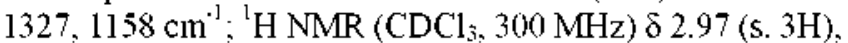
3.69 (s. $3 \mathrm{H}$ ). 4.09 (d. $J=6.3 \mathrm{~Hz}, 2 \mathrm{H}$ ). 5.09 (br s. IH). 7.03 (s. lH). 7.26-7.34 (m, 5H): ${ }^{13} \mathrm{C} \mathrm{NMR}\left(\mathrm{CDCl}_{3}, 75 \mathrm{MHz}\right) \delta 41.49$. $47.42,51.87,128.18,128.53 .128 .60,128.78,134.61$. 138.91, 168.03

Typical procedure for the synthesis of $3 \mathrm{~d}$. A solution of $\mathbf{1 h}$ (159 mg. $\mathrm{l} \mathrm{mmol})$. $\mathrm{TsNH}_{2}(257 \mathrm{mg} .1 .5 \mathrm{mmol})$, and $\mathrm{CH}_{2} \mathrm{SO}_{3} \mathrm{H}$ $(1.2 \mathrm{~mL})$ in 1,2 -dichloroethane $(5 \mathrm{~mL})$ was heated to reflux for $2 \mathrm{lh}$. After usual aqueous workup and colunun chromatographic purification process (hexanes/EtOAc/CH$/ \mathrm{Cl}_{2}$. $3: 1: 3$ ) we obtained $3 \mathrm{~d}-Z$ (216 $\mathrm{mg}$. $69 \%$ ) as a white solid. The other compounds were synthesized analogously and the spectroscopic data of $\mathbf{3 ( \mathrm { l } - Z} Z$. 3e- $Z$. and $+(Z Z Z)$ are as follows.

Compound 3d-Z: $69 \%$ : white solid mp $147-149^{\circ} \mathrm{C}$ : IR (film) 3268, 2213.1328, $1159 \mathrm{~cm}^{-1}:{ }^{1} \mathrm{H}$ NMR (CDClㅅ․ 300 $\mathrm{MHz}) \hat{o} 2.34$ (s. $3 \mathrm{H}), 3.95$ (d. $J=6.6 \mathrm{~Hz} .2 \mathrm{H}) .5 .16(\mathrm{t} . J=6.6$ Hz. lH). 7.02 (s. lH). 7.25 (d. $J=8.4 \mathrm{~Hz}, 2 \mathrm{H}) .7 .36-7.4 \mathrm{l}(\mathrm{m}$. $3 \mathrm{H}) .7 .56-7.60(\mathrm{~m}, 2 \mathrm{H}), 7.76(\mathrm{~d}, J=8.4 \mathrm{~Hz}, 2 \mathrm{H}) ;{ }^{13} \mathrm{C} \mathrm{NMR}$ $\left(\mathrm{CDCl}_{3}, 75 \mathrm{MHz}\right)$ ò $21.43,47.03,106.38,117.24,127.25$. $128.82,128.88,129.84 .130 .84,132.50,137.01 .143 .98,145.63$.

Compound 3e-Z: 71\%: white solid. np 103-104 ${ }^{\circ} \mathrm{C}$ (decomp.): IR (film) 3343, 2213,1312.1144 $\mathrm{cm}^{-1}:{ }^{1} \mathrm{H}$ NMR $\left(\mathrm{CDCl}_{3} .300 \mathrm{MHz}\right)$ ô $3.06(\mathrm{~s} .3 \mathrm{H}), 4.13(\mathrm{~d}, J=6.3 \mathrm{~Hz}, 2 \mathrm{H})$, 4.75 (br s. $\mathrm{lH}) .7 .20$ (s. $\mathrm{lH}) .7 .45-7.47(\mathrm{~m} .3 \mathrm{H}) .7 .76-7.79(\mathrm{~m}$. $2 \mathrm{H}) ;{ }^{13} \mathrm{C} \mathrm{NMR}\left(\mathrm{CDCl}_{3}, 75 \mathrm{MHz}\right) \delta+2.36,47.28 .107 .02$, $117.52,129.06(2 \mathrm{C}), 131.20,132.39,146.09$

Compound 4: $7 \%$ : white solid, mp $160-162^{\circ} \mathrm{C}$ : IR (film) $2215,1349,1161.1092 \mathrm{~cm}^{-1}:{ }^{1} \mathrm{H}$ NMR $\left(\mathrm{CDCl}_{5}, 300 \mathrm{MHz}\right) \delta$ 2.38 (s. $3 \mathrm{H}) .+29$ (s. $+\mathrm{H}) .7 .23$ (s. $2 \mathrm{H}) .7 .28$ (d. $J=8.5 \mathrm{~Hz}$. $2 \mathrm{H}) .7 .38-7.43$ (m. $6 \mathrm{H}) .7 .67-7.70(\mathrm{~m} .+\mathrm{H}) .7 .76(\mathrm{~d}, J=8.5$ $\mathrm{Hz}, 2 \mathrm{H}):{ }^{13} \mathrm{C} \mathrm{NMR}\left(\mathrm{CDCl}_{3}, 75 \mathrm{MHz}\right) \hat{\delta} 21.50,51.72,105.33$. $117.61,127.58 .128 .90,129.13 .129 .96,131.07$. 132.46, 136.33, $1+4.36 .147 .42$.

\section{References and Notes}

1. For the general review on Baylis-Hillman reaction, see: (a) Basavaiah, D.; Rao, A. I.; Satyanarayana, T. Chem. Rev. 2003. 103,811-891. (b) Singh, V; Batra, S. Tetrahedron 2008, 64, 4511-4574. (c) Ciganek, E. In Organic Reactions; Paquette, L. A., Ed.; Joln Wiley \& Sons: New York, 1997; Vol. 51, pp 201-350. (d) Basavaiah. D.: Rao. P. D.: Hyma, R. S. Tetrahedron 1996, 52,8001-8062 (e) Drewes, S. E.; Roos, G. H. P. Tetrahedron 1988, 44,4653-4670. (f) Kim, J. N.; Lee, K. Y. Cum Oig. Chem. 2002, 6, 627-645. (g) Lee, K. Y.; Gowrisankar, S.; Kim, J. N. Bull. Korean Chem. Soc. 2005, 26, 1481-1490. (h) Langer, P. Angew: Chem. Int Ed 2000, 39, 3049-3052. (i) Krishna, P. R.; Sachwani, R.: Reddy, P. S. Sinlett 2008, 2897-2912. (j) Declerck, 
V.; Martinez, T.; Lamaty, F. Chem. Rev, 2009, 109, 1-48.

2. For the synthesis of rearranged $N$-tosyl aza-Baylis-Hillnann adducts, see: (a) Kinn, J. N.; Chung, Y. M.; In, Y. J. Tetrahedron Lett. 2002, 43,6209-6211. (b) Xu, Y.-M.; Shi, M. J. Org. Chem. 2004, 69, 417-425. (c) Ciclosi. M.: Fava, C.: Galeazzi, R.: Orena, M.: Sepulveda-Arques, I. Tetrahetron Lett. 2002, 13 , 2199-2202.

3. For the synthetic applications of K-tosyl $a z a$-Baylis-Hillman adducts and their rearranged derivatives, see: (a) Park, D. Y:; Lee, M. T.; Kin, T. H.; Kim, .. N. Tetrahedron Lett. 2005, 16 , 8799-8803. (b) Gowrisankar, S.: Lee, K. Y.: Kim, T. H.: Kim, J N. Tetrahedron Lett. 2006, 47,5785-5788, (c) Lee, M. T.; Kim, S. C.: Kim. J. N. Bull. Korean Chen. Soc. 2006, 27, 439-442. (d) Kim, I. M: Lee, K. Y: Lee, S.: Kim, I. N. Tetrahedron Lett. 2004, 15, 2805-2808. (e) Kim, I. N.: Lee, H. I: Lee, K. Y.; Kin, H. S. Tetrahedron Lett. 2001, 42,3737-3740. (f) Chnng, Y. M.: Lee, H. J.; Hwang, S. S : Kim, J. N. Bull. Korean Chem. Soc. 2001, 22,799-800. (g) Lee, H. S.; Kim, J. M; Kim, I. N. Tetrahed

4. For our recent papers on radical cyclization involving modified Bavlis-Hillman adducts, see: (a) Gowrisankar, S.; Kim, S. J.; Lee, J.-E.: Kim, J. N. Tetrahedron Lent 2007, 18, 4419-4422. (b) Gowrisankar, S.: Lee. H. S.: Kim. I. N. Tetrahedron Lett. 2007. 18, 3105-3108. (c) Gowrisankar, S.: Lee, K. Y.: Kim, T. H.: Kim, J. N. Tetrahedron Lett. 2006, $17,5785-5788$. (d) Gowrisankar, S.; Lee, H. S.; Kim, J. N. Bull. Korean Chem. Soc, 2006, 27, 2097-2100. (e) Gowrisankar, S.; Lee, K. Y: Kim, J. N. Bull.
Kolean Chem. Soc. 2006, 27, 929-932. (f) Park. D. Y.: Gowrisankar, S.: Kim, J. N. Bull. Konean Chem. Soc. 2005, 26, 1410-1442. (g) Gowrisankar. S.: Lee. K. Y.: Kim. I. N. Tetrahedron Letr. 2005, 46, 4859-4863. (h) Lee, H. S.; Kim, H. S.; Kim, J. M.; Kim, J. N. Tetrahedron 2008, 6t, 2397-2404 and further references cited therein

5. (a) Gowrisankar. S.: Lee, H. S.: Kim. J. M.: Kim. I. N. Tetrhitedron Lett. 2008, $99,1670-1673$. (b) Lee, H. S.; Kim, S. H.: Gotwisarkar, S.; Kim, J. N. Tetrahedron 2008, 64, 7183-7190. (c) Gowrisankar, S.; Lee, H. S.: Lee, K. Y.: Lee, I.-E.: Kim, I. N. Tetrahedron Lett. 2007, 48. 8619-8622.

6. For the recent applications of montmorillonite $\mathrm{K} 10$ in organic synthesis, see: (a) Shanmugam, P.; Rajasingh, P. Tetrohedron 2004, 60, 9283-9295. (b) Shanmugam, P.: Rajasingh, P. Chem. Lett. 2005. 34. 1494-1495. (c) Shanmugam. P. Singh. P. R. Synlett 2001, 1314-1316. (d) Das, B.: Majhi, A.; Baneriee, I.; Chowdhur, N.; Venkateswarlu, K. Chem. Lett. 2005, 3f, 14921493

7. The isomerization of $3 \mathbf{b}-E$ to $3 \mathbf{b}-2$ did not occur at all in the presence of $\mathrm{TSNH}_{2} / \mathrm{K} 10$. however. we observed that $3 \mathrm{~b}-Z$ isomerized into $3 \mathbf{b}-E$ slowly under the same conditions.

8. Three isomers having $E E, E: Z$, and $Z Z Z$ configurations were mised together. These types of bis-adducts have been reported. see: (a) Pathak. R.: Singh. V: Nag. S. N.: Kanojiva, S.: Batra. S. Symthesis 2006, 813-816. (b) Singh, V.; Pathak, R.; Kanojiya, S.; Batra, S. Sntlet 2005, 2465-2468. (c) Basavaiah. D.; Satyanaravana, T. Org. Lett. 2001, 3, 3619-3622. 